\title{
Justesse de la technique des films secs réhydratables (Petrifilm SM et VRB) pour la numération des flores totale, psychrotrophe et coliforme du lait cru à l'usine
}

\author{
C Piton, D Rongvaux-Gaïda
}

INRA, station de recherches en technologie et analyses laitières, BP 89, 39800 Poligny, France

(Reçu le 29 janvier 1990; accepté le 15 mars 1990)

\begin{abstract}
Résumé - Cent quarante échantillons de lait cru de citernes de ramassage de 4 entreprises laitières contenant entre $10^{4}$ et $6,6 \times 10^{7} \mathrm{UFC} / \mathrm{ml}$ ont été soumis à la numération de la flore totale sur Petrifilm SM à $30^{\circ} \mathrm{C}$, des bactéries coliformes sur Petrifilm VRB à $30^{\circ} \mathrm{C}$ et de la flore psychrotrophe sur Petrifilm SM après $48 \mathrm{~h}$ d'incubation à $21^{\circ} \mathrm{C}$ (technique modifiée d'Oliveria et Parmelee). Ces 3 groupes microbiens ont également été dénombrés selon les techniques de référence à l'aide de l'ensemenceur Spiral. Pour la flore totale, l'équation de la régression Référence sur Petrifilm est log (Réf) $=0,991 \times \log$ (Petrifilm) $+0,021$, avec un coefficient de corrélation de 0,985 et un écart type résiduel de $0,128 \log (\mathrm{UFC} / \mathrm{ml})$; la pente et l'ordonnée à l'origine de la droite ne sont pas significativement différentes respectivement de 1,000 et de 0,000 au seuil de $5 \%$. Pour les bactéries coliformes, l'équation de la régression est $\log$ (Réf) $=0,891 \log$ (Petrifilm) $+0,471$, avec un coefficient de corrélation de 0,934 et un écart type résiduel de $0,250 \log$ (UFC/ml); la pente et l'ordonnée à l'origine de la droite sont significativement différentes respectivement de 1,000 et de 0,000 au seuil de $1 \%$ et le Petrifilm VRB sous-estime les nombres de bactéries coliformes en moyenne de $37,2 \%$. Enfin, pour la flore psychrotrophe, l'équation de la régression Référence $7^{\circ} \mathrm{C}-10 \mathrm{j}$ sur Petrifilm SM $21^{\circ} \mathrm{C}-48$ h est $\log ($ Ref $)=1,029 \times \log ($ Petrifilm) $-0,258$, avec un coefficient de corrélation de 0,985 et un écart type résiduel de $0,145 \mathrm{log}$ (UFC/ml); la pente n'est pas significativement différente de 1,000 au seuil de $5 \%$ mais les nombres d'UFC/ml obtenus sur Petrifilm sont en moyenne supérieurs de $26 \%$ aux nombres de bactéries psychrotrophes obtenus selon la technique de référence. La justesse de cette méthode rapide de numération de la flore psychrotrophe sur Petrifilm semble influencée par la nature de la flore microbienne du lait.
\end{abstract}

film sec réhydratable / Petrifilm / flore totale / flore psychrotrophe / coliforme / lait cru

Summary - Accuracy of the dry rehydratable film methods (Petrifilm SM and VRB) for the enumeration of total, psychrotrophic and coliform bacteria in raw milk at the dairy plant. One hundred and forty bulked tanker milk samples ranging between $10^{4}$ and $6.6 \cdot 10^{7} \mathrm{CFU} / \mathrm{ml}$ were submitted to the enumeration of total flora on Petrifilm SM at $30^{\circ} \mathrm{C}$, coliform bacteria on Petrifilm VRB at $30{ }^{\circ} \mathrm{C}$ and psychrotrophic flora on Petrifilm SM after $48 \mathrm{~h}$ of incubation at $21{ }^{\circ} \mathrm{C}$. They were also analysed according to the reference techniques using the spiral plate maker. For total flora, the equation of the regression line Reference on Petrifilm is: $\log ($ Ref $)=0.991 \times \log ($ Petrifilm $)+0.021$, with a correlation coefficient of 0.985 and a residual standard deviation of $0.128 \mathrm{log}(\mathrm{CFU} / \mathrm{ml})$; the slope and the intercept of the regression line do not differ significantly from 1.000 and 0.000 respectively at the $5 \%$ level. For coliform bacteria, the equation of the regression line is: $\log ($ Ref) $=0.891 \times \log$ (Petrifilm) +0.471 , with a correlation coefficient of 0.934 and a residual standard deviation of $0.250 \mathrm{log}$ (CFU/ml); the slope and the intercept of the line differ respectively from 1.000 and 0.000 at the $1 \%$ level and the Petrifilm counts are significantly lower than the reference counts by $37.2 \%$ on average. 
For the psychrotrophic flora, the equation of the regression line Reference $7^{\circ} \mathrm{C}-10 \mathrm{~d}$ on Petrifilm $21^{\circ} \mathrm{C}-48 \mathrm{~h}$ is $: \log ($ Ref $)=1.029 \log$ (Petrifilm) -0.258 , with a coefficient of correlation of 0.985 and a residual standard deviation of $0.145 \mathrm{log}(\mathrm{CFU} / \mathrm{ml})$; the slope of the regression line does not differ significantly from 1.000 at the $5 \%$ level but the Petrifilm counts are significantly higher than the reference counts by $26 \%$ on average. The accuracy of this rapid method for the enumeration of the psychrotrophic flora seems to be influenced by the nature of the milk microbial flora.

dry rehydratable film method / Petrifilm / total flora / psychrotrophic flora / coliform / raw milk

\section{INTRODUCTION}

Depuis quelques années, la technique des films secs réhydratables (Petrifilm SM et VRB; marque déposée 3M Biomédical) s'est développée pour remplacer les techniques de référence, respectivement pour le dénombrement de la flore totale et des bactéries coliformes dans les produits alimentaires. Le Petrifilm est constitué d'un double film enduit de produit déshydratés : le film inférieur sert de support au milieu de culture (PCA ou VRBA) et à un agent gélifiant soluble dans l'eau froide, tandis que le film supérieur est enduit de l'agent gélifiant et d'un indicateur au tétrazolium qui donne une coloration rouge aux colonies. Dans le cas du Petrifilm VRB, la fermentation du lactose par les coliformes s'accompagne d'une production de gaz : ce gaz est piégé par le film supérieur et s'accumule autour des colonies, ce qui permet de les différencier de celles des autres micro-organismes à Gram négatif.

La technique du film sec réhydratable présente de nombreux avantages : gain de temps (pas de préparation de milieux), faible encombrement et facilité de stockage. D'autre part, pour le lait cru, sa fidélité est désormais bien établie, à la fois pour la numération de la flore totale et celle des bactéries coliformes (Ginn et al, 1986; Piton et Grappin, 1990). Ainsi, Piton et Grappin (1990) ont pu montrer que ses caractéristiques de répétabilité et de reproductibilité sont équivalentes, voire meilleures dans le cas de la numération des coliformes, que celles des techniques de référence.

De nombreux travaux ont déjà clairement démontré que cette technique présente également une relation étroite avec les méthodes de référence, avec cependant une sous-estimation du nombre de colonies pour la flore totale (tableaux I et II). Les coefficients de corrélation avec la technique de référence sont supérieurs à 0,95 dans le cas du Petrifilm SM ou à 0,93 dans le cas du Petrifilm VRB. Mais le plus souvent, les auteurs ne précisent pas l'ensemble des paramètres de la justesse (précision d'estimation et exactitude de calibrage); or, la valeur du coefficient de corrélation est largement influencée par l'étendue des niveaux de contamination des échantillons analysés.

Par ailleurs, Bishop et Juan (1988) ont tenté d'appliquer au Petrifilm SM la technique de dénombrement de la flore psychrotrophe décrite par Oliveria et Parmelee (1976) : celle-ci consiste en une numération des colonies sur milieu Plate Count Agar, après $25 \mathrm{~h}$ d'incubation à $21^{\circ} \mathrm{C}$. Bishop et Juan (1988) ont noté, sur Petrifilm $S M$, une relation plus étroite entre la technique de référence et celle d'Oliveria et Parmelee et un calibrage plus satisfaisant, qu'en boîte de Petri classique; ils ont attribué ce fait à un comptage plus aisé des colonies sur Petrifilm, en raison de l'incorporation de triphényl tétrazolium chlorure (TTC) dans le milieu. Toutefois, là encore, 
les auteurs ne donnent ni l'étendue des niveaux de contamination des échantillons, ni la précision d'estimation de la technique.

L'objectif de cette étude a donc été d'établir expérimentalement tous les paramètres de la justesse des films secs réhydratables, pour la numération de la flore totale, des bactéries coliformes et de la flore psychrotrophe du lait cru. L'étude a porté sur une population d'échantillons prélevés dans des citernes de ramassage d'entreprises laitières, à l'arrivée à l'usine.

\section{MATÉRIEL ET MÉTHODES}

\section{Origine des échantillons}

Cent quarante échantillons de lait cru ont été prélevés dans des citernes de ramassage de 4 entreprises laitières. Ils ont été conservés à $0^{\circ} \mathrm{C}$, au plus $24 \mathrm{~h}$ après le prélèvement.

\section{Numérations microbiennes}

Avant la numération, les échantillons de lait cru ont subi une agitation pendant $30 \mathrm{~s}$ à l'aide d'un homogénéisateur à turbine (Ultra-turrax, type $18 / 10$ ) tournant à 20000 tours $/ \mathrm{min}$. Ce mode d'agitation est actuellement celui qui assure la meilleure dispersion des amas microbiens (Richard, 1980; Richard et al, 1981).

Les échantillons agités ont ensuite été soumis au dénombrement des 3 groupes microbiens suivants :

- flore aérobie mésophile sur milieu Plate Count Agar (Difco), après 3 j d'incubation à $30^{\circ} \mathrm{C}$;

- bactéries coliformes sur milieu Violet Red Bile Agar (Difco) après $24 \mathrm{~h}$ d'incubation à $30^{\circ} \mathrm{C}$;

- flore psychrotrophe sur milieu Plate Count Agar (Difco), après $10 \mathrm{j}$ d'incubation à $7{ }^{\circ} \mathrm{C}$.

Les ensemencements ont été réalisés à la surface de la gélose à l'aide de l'appareil Spiral décrit par Gilchrist et al (1973). Nous avons choisi cette méthode comme technique de réfé- rence car il a largement été montré qu'elle donne des résultats similaires à ceux de la technique classique de dénombrement (Campbell et Gilchrist, 1973; Gilchrist et al, 1973; Donnelly et al, 1976; Jarvis et al, 1977; Peeler et al, 1977). D'autre part, contrairement à ce qui est recommandé pour la numération des bactéries coliformes (Anonyme, 1985a), nous n'avons pas recouvert le milieu ensemencé d'une seconde couche de VRB, pour inhiber les microorganismes aérobies stricts qui peuvent se développer sur ce milieu. En effet, d'après Richard et al (1988), la flore lactose-positive sur VRBA peut être identifiée à des entérobactéries dans environ $95 \%$ des cas et le développement des colonies lactose-négatives n'affecte pas la croissance des colonies de bactéries coliformes.

Chacun des échantillons agités a également été soumis au dénombrement:

- de la flore aérobie mésophile après 72 h d'incubation à $30^{\circ} \mathrm{C}$ sur Petrifilm SM, selon les recommandations du fournisseur;

- des bactéries coliformes sur Petrifilm VRB, après incubation à $30^{\circ} \mathrm{C}$ et selon les recommandations du fournisseur. Nous avons cependant allongé la durée d'incubation à $36-48 \mathrm{~h}$, pour favoriser la production de gaz chez les colonies de bactéries coliformes faibles productrices (Nelson et al, 1984);

- de la flore psychrotrophe sur Petrifilm SM, selon la technique d'Oliveria et Parmelee (1976), c'est-à-dire après une incubation à $21^{\circ} \mathrm{C}$. Par rapport à la technique originale, nous avons allongé la durée de l'incubation qui a été portée de 25 à $48 \mathrm{~h}$, car l'incorporation de TTC au milieu semble ralentir la croissance microbienne (Bishop et Juan, 1988).

Pour les numérations sur Petrifilm SM, un Petrifilm a été ensemencé à partir de chacune des dilutions $-3,-4$ et -5 ; dans le cas des coliformes, un Petrifilm VRB a été ensemencé a partir de chacune des dilutions $-1,-2$ et -3 . $\dot{A}$ l'issue de la période d'incubation, les colonies ont été comptées sur la totalité de la surface du Petrifilm. Les Petrifilms SM contenant plus de 400 colonies ont été déclarés incomptables; il en a été de même pour les Petrifilms VRB contenant plus de 100 colonies productrices de gaz. Le nombre d'unités formant colonies par millilitre de lait (UFC/ml) a ensuite été estimé pour chaque échantillon à partir de la formule suivante: 


$$
N=\frac{\sum C_{i}}{\Sigma V_{i}}
$$

$C_{i}$ et $V_{i}$ étant respectivement le nombre de colonies et le volume d'échantillon pour le Petrifilm de la dilution $i$.

Chaque échantillon a été analysé en double par chaque technique, afin d'obtenir une meilleure précision des résultats de numération.

\section{Calculs statistiques}

La justesse de la technique du film sec réhydratable a été calculée conformément aux recommandations de la norme FIL 128 (Anonyme, 1985b); avant les calculs statistiques, les nombres d'UFC/ml ont subi une transformation logarithmique destinée à normaliser les distributions.

La justesse représente l'étroitesse de l'accord entre la valeur vraie de la grandeur à mesurer (par convention, le nombre d'UFC/ml obtenu selon les techniques de référence) et le résultat moyen qui serait obtenu en appliquant la technique expérimentale un grand nombre de fois (Anonyme, 1985b). La justesse d'une technique dépend des 2 notions suivantes :

\section{Exactitude de calibrage}

Conformément à la norme FIL, l'équation de calibrage $(y=b x+a)$ a été établie selon la méthode des moindres carrés à partir des moyennes des 2 déterminations faites sur chaque échantillon pour les 2 techniques. Les logarithmes décimaux des nombres d'UFC/ml obtenus avec l'appareil Spiral sont considérés comme variable dépendante et les logarithmes décimaux des nombres d'UFC/ml obtenus sur film sec réhydratable comme variable indépendante, pour donner une estimation de l'écart type résiduel dans l'unité de la technique de référence. II faut noter que la norme FIL a été établie pour les méthodes chimiques et que certaines hypothèses ne sont plus vérifiées dans le cas des techniques microbiologiques : en particulier, le calcul du coefficient de régression et de l'écart type résiduel suppose une erreur négligeable dans la mesure des $x$ et des $y$, ce qui n'est pas le cas de ces techniques. Pour que la méthode soit exactement calibrée, il faut que le coefficient de régression ne soit pas statistiquement différent de 1 et que l'ordonnée à l'origine ne soit pas différente de 0 , cette dernière condition pouvant être remplacée par l'égalité des moyennes des 2 variables.

\section{Précision d'estimation}

La précision d'estimation de la technique expérimentale est donnée par les limites de l'intervalle de confiance à l'intérieur duquel se situe, avec une probabilité de $95 \%$, la vraie valeur donnée par la méthode de référence. Si $y$ est le nombre d'UFC/ml calculé à partir de la droite de calibrage, ces limites sont, en valeurs logarithmiques, $\log y \pm 1,96 s_{y, x}$ où $s_{y, x}$ est l'écart type résiduel des écarts à la droité de régression. En données non transformées, ces limites sont $y$ / $10^{1,96 s_{y, x}}$ pour la limite inférieure et $y \times 10^{1,96 s_{y, x}}$ pour la limite supérieure.

\section{RÉSULTATS}

\section{Flore totale}

La figure 1 montre une relation très étroite entre les logarithmes des nombres d'UFC/ $\mathrm{ml}$ obtenus selon la technique de référence et sur film sec réhydratable (Petrifilm SM) sur la population des 140 échantillons analysés. L'équation de calibrage a été calculée après élimination de 2 échantillons qui s'écartent de plus de 3 écarts types résiduels de cette droite. La précision d'estimation du film $\mathrm{sec}$ réhydratable pour la numération de la flore totale est donc, en valeurs logarithmiques, de $\pm 1,96$ $x 0,128$, soit $\pm 0,251 \log (\mathrm{UFC} / \mathrm{ml})$. Si $y$ est le nombre d'UFC/ml estimé à partir de la droite de calibrage, les limites de l'intervalle de confiance de $y$ (à $P=0,95)$ sont, en données non transformées :

$y / 100,251$, soit $y / 1,78$ pour la limite inférieure et $y \times 10^{0,251}$, soit $y \times 1,78$ pour la limite supérieure. 
La pente et l'ordonnée à l'origine de la droite de calibrage ne sont pas significativement différentes respectivement de 1,000 et de 0,000 au seuil de $5 \%$. Par conséquent, le film sec réhydratable est une technique parfaitement calibrée et les résultats peuvent être utilisés directement sans avoir recours à un facteur de correction. La différence entre les 2 moyennes $(\log ($ Réf $)=5,472$ et $\log ($ Petrifilm $)=5,500$ ) est par ailleurs assez faible $(+6,7 \%$ en $\mathrm{UFC} / \mathrm{ml})$.

\section{Bactéries coliformes}

Sur la figure 2, est présentée la relation entre les logarithmes des nombres de coli- formes obtenus sur VRBA et sur film sec réhydratable (Petrifilm VRB). La relation a été établie sur urie population de 137 échantillons; en effet, nous avons dû éliminer 3 échantillons car les dilutions choisies n'étaient pas assez fortes pour permettre un comptage précis des colonies productrices de gaz sur film sec réhydratable. Tout d'abord, on observe une dispersion beaucoup plus importante des points autour de la droite que dans le cas de la flore totale. Pour la numération des bactéries coliformes, la précision d'estimation du film sec réhydratable est de $\pm 1,96 \times 0,250$, soit $\pm 0,490 \log (\mathrm{UFC} / \mathrm{ml})$. Si $y$ est le nombre de coliformes estimé à partir de la droite de calibrage, les limites de l'intervalle de confiance de y (à $P=0,95)$ sont, en données non transformées :

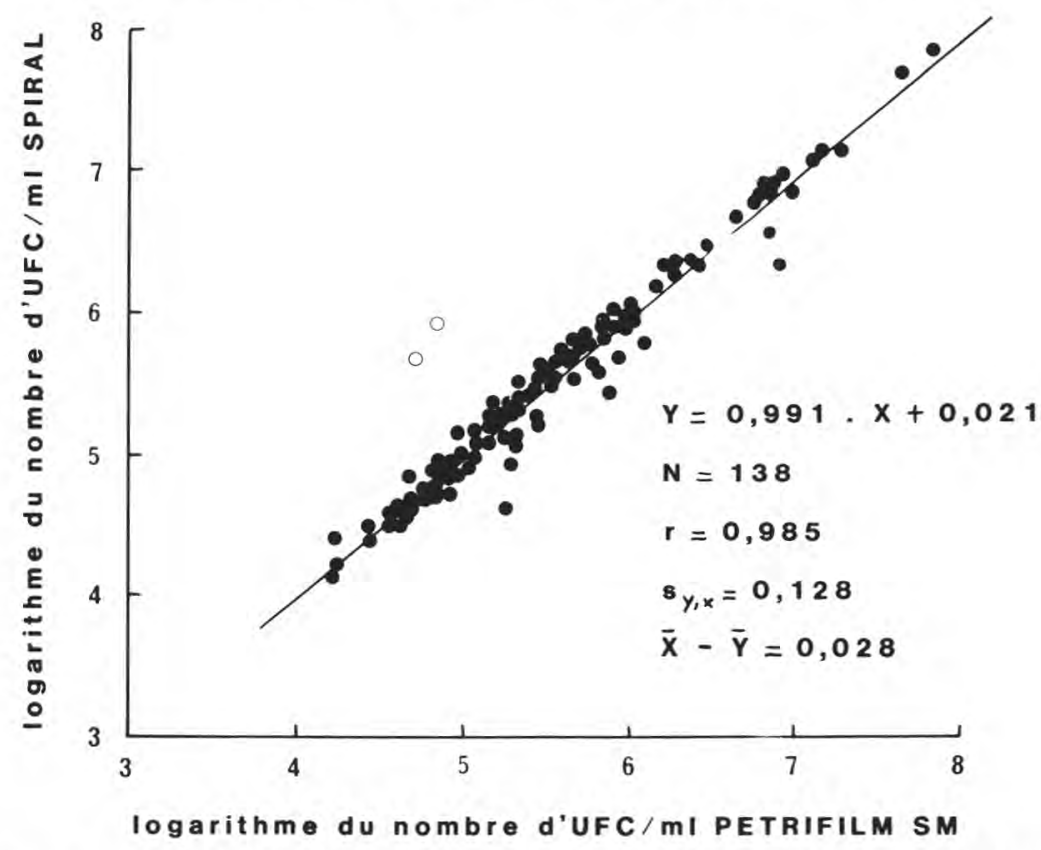

Fig 1. Relation entre les logarithmes décimaux des nombres d'UFC/ml obtenus selon la technique de référence Spiral et sur film sec réhydratable (Petrifilm SM), sur une population de 140 échantillons de lait cru prélevés à l'usine. L'équation de la droite de calibrage a été calculée après élimination de 2 échantillons $(\mathrm{O})$ qui s'écartent de plus de 3 écarts types résiduels de cette droite.

Relationship between the logarithms of the numbers of CFU/ml obtained with the reference technique and with the dry rehydratable film method (Petrifilm SM). 
Tableau I. Justesse de la technique du film sec réhydratable (Petrifilm SM) pour la numération de la flore totale du lait cru.

Accuracy of the dry rehydratable film method (Petrifilm SM) for the enumeration of total flora in raw milk.

\begin{tabular}{|c|c|c|c|c|c|}
\hline \multirow[b]{2}{*}{ Références } & \multicolumn{5}{|c|}{ Paramètres statistiques } \\
\hline & Étendue log (UFC) & Équation de régression & $r$ & $s_{y, x}$ & $\bar{X}-\bar{Y}$ \\
\hline Ginn et al (1984) & $3-7$ & $Y=0,997 X+0,191$ & 0,946 & - & $-0,177$ \\
\hline Dasen et Duboz (1985) & $3-7$ & $Y=0,901 X+0,694$ & 0,973 & 0,165 & $-0,188$ \\
\hline Hughes et Sutherland (1987) & $3,5-5,5$ & $Y=0,962 X+0,337$ & 0,946 & - & - \\
\hline Bishop et Juan (1988) & - & $Y=0,998 X+0,117$ & 0,971 & - & $-0,110$ \\
\hline
\end{tabular}

$X$ : logarithme du nombre d'UFC/ml Petrifilm; $Y$ : logarithme du nombre d'UFC/ml Technique de référence; $r$ : coefficient de corrélation; $s_{y, X}$ : écart type résiduel (en log UFC/ml); $\bar{X}-\bar{Y}:$ différence entre les moyennes des $X$ et des $Y$ (en $\log \mathrm{UFC} / \mathrm{ml}) ;(-)$ : non déterminé ou précisé par les auteurs.

$\mathrm{X}$ : logarithm of the number of CFU/mI Petrifilm; Y: logarithm of the number of CFU/mI Reference technique; $\mathrm{r}$ : correlation coefficient; $\mathrm{s}_{\mathrm{y}, \mathrm{X}}$ : residual standard deviation of the regression in $\log (C F U / \mathrm{ml}) ; \overline{\mathrm{X}}-\overline{\mathrm{Y}}$ : difference between the means $\bar{X}$ and $\bar{Y}$ in log (CFU/ml); (-): not determined or precised.

$y / 10^{0,490}$, soit $y / 3,09$ pour la limite inférieure

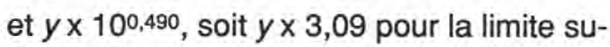
périeure.

La pente et l'ordonnée à l'origine de la droite de calibrage sont significativement différentes respectivement de 1,000 et de 0,000 au seuil de $1 \%$; de même, les moyennes log (Réf) $=3,312$ et log (Petrifilm) $=3,189$, sont significativement différentes au seuil de $1 \%$. Cela signifie que pour la numération des bactéries coliformes, le film sec réhydratable n'est pas une technique correctement calibrée et qu'il est nécessaire d'appliquer l'équation de calibrage aux résultats donnés par cette technique pour estimer le nombre de coliformes dans l'échantillon. La différence moyenne entre les résultats des 2 techniques est de $-0,123 \log (\mathrm{UFC} / \mathrm{ml})$, c'est-àdire de $-32,7 \%$ en données non transformées.

\section{Flore psychrotrophe}

La figure 3 présente la relation entre les logarithmes des nombres de bactéries psychrotrophes obtenus selon la technique de référence $\left(7^{\circ} \mathrm{C}\right.$ pendant $\left.10 \mathrm{j}\right)$ et les logarithmes de ces nombres obtenus sur film sec réhydratable (Petrifilm SM) après $48 \mathrm{~h}$ d'incubation à $21^{\circ} \mathrm{C}$. La droite de calibrage a été calculée après élimination de 4 échantillons qui s'écartaient de plus de 3 écarts types résiduels de cette droite. La précision d'estimation de cette technique est de $\pm 1,96 \times 0,145$, soit $\pm 0,284 \mathrm{log}$ (UFC/ml). Si $y$ est le nombre d'UFC/ml estimé à partir de la droite de calibrage, les limites de l'intervalle de confiance de $y$ (à $P$ $=0,95$ ) sont, en données non transformées:

$y / 10^{0,284}$, soit $y / 1,92$ pour la limite inférieure et $y \times 10^{0,284}$, soit $y \times 1,92$ pour la limite supérieure. 


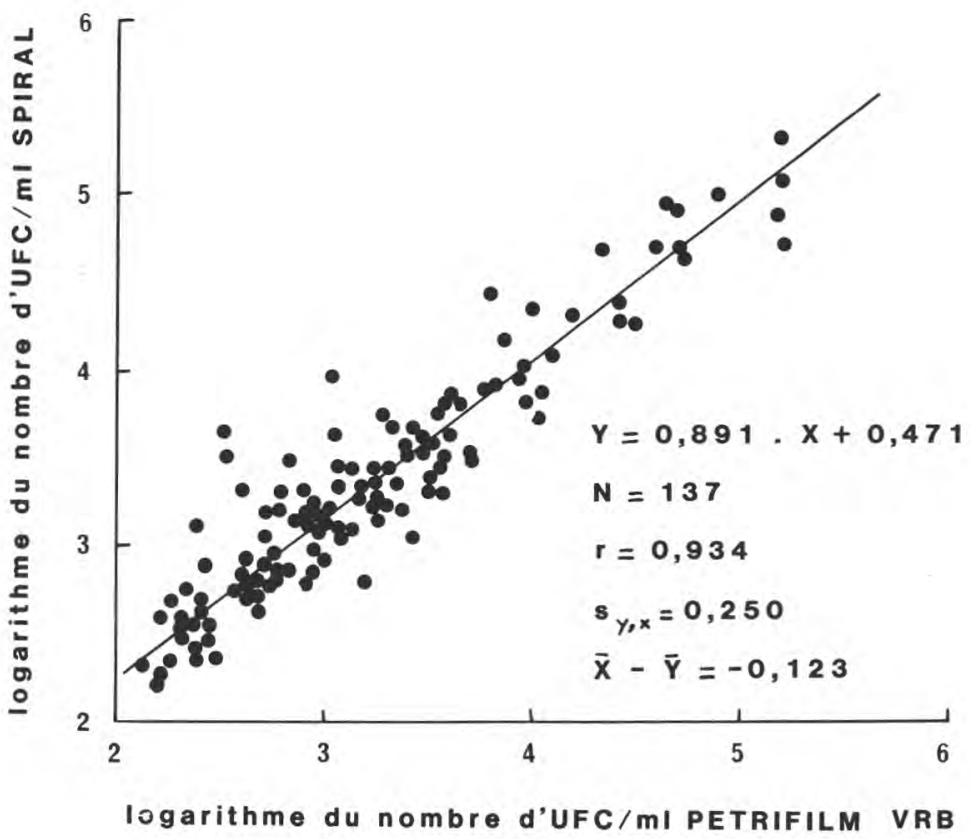

Fig 2. Relation entre les logarithmes décimaux des nombres de coliformes obtenus sur VRBA selon la technique Spiral et sur film sec réhydratable (Petrifilm VRB), sur une population de 137 échantillons de lait cru prélevés à l'usine.

Relationship between the logarithms of the numbers of coliforms obtained with the reference technique and with the dry rehydratable method (Petrifilm VRB).

La pente de la droite de calibrage n'est pas significativement différente de 1,000 au seuil de $5 \%$. Par contre, l'ordonnée à l'origine de cette droite est significativement différente de 0,000 au seuil de $1 \%$; il en est de même des moyennes log (Réf) = 5,337 et $\log ($ Petrifilm $)=5,437$. Cela signifie que la technique n'est pas correctement calibrée et qu'il faut appliquer un facteur de correction aux résultats obtenus sur film sec réhydratable. Ce facteur de correction est égal à $10^{\bar{x}} \bar{Y}$, soit $10^{0,100}=1,26$ : il suffit donc de diviser les résultats obtenus sur film sec réhydratable par 1,26 pour obtenir les nombres réels de bactéries psychrotrophes.

Enfin, nous avons tenté d'analyser les causes des écarts importants de 4 échantillons à la droite de calibrage. Deux des 3 échantillons au-dessus de la courbe (nette sous-estimation avec la technique expérimentale) correspondent à des laits contenant une flore psychrophile dominante incapable de croître sur PCA à 21 ou $30^{\circ} \mathrm{C}$; l'échantillon au-dessous de la courbe (nette surestimation avec la technique expérimentale) correspond à un lait conte- 
Tableau II. Justesse de la technique du film sec réhydratable (Petrifilm VRB) pour la numération des bactéries coliformes du lait cru.

Accuracy of the dry rehydratable film method (Petrifilm VRB) for the enumeration of coliform bacteria in raw milk.

Paramètres statistiques

Référence Étendue log (UFC) Équation de régression $\begin{array}{rlll}r & \mathrm{~s}_{\mathrm{y}, \mathrm{x}} & \overline{\mathrm{X}}-\overline{\mathrm{Y}}\end{array}$

$\begin{array}{lccccc}\text { Nelson et al (1984) } & 0,5-4,5 & Y=1,042 X+0,079 & 0,932 & - & - \\ \text { Branger (1987) } & 1-6 & Y=0,923 X+0,608 & 0,978 & 0,281 & -0,334 \\ \text { Bishop et Juan (1988) } & - & Y=0,983 X-0,024 & 0,963 & - & 0,054\end{array}$

$X$ : logarithme du nombre d'UFC/ml Petrifilm; $Y$ : logarithme du nombre d'UFC/ml Technique de référence; $r$ : coefficient de corrélation; $s_{y, x}$ : écart type résiduel (en log UFC/ml); $\bar{X}-\bar{Y}$ : différence entre les moyennes des $X$ et des $Y$ (en log UFC/ml); (-): non déterminé ou précisé par les auteurs.

$\mathrm{X}$ : logarithm of the number of CFUiml Petrifilm; Y: logarithm of the number of CFU/ml Reference technique; $r$ : correlation coefficient; $\mathrm{s}_{\mathrm{y}, \mathrm{x}}$ : residual standard deviation of the regression in log (CFU/mI); $\overline{\mathrm{X}}-\overline{\mathrm{Y}}:$ difference between the means $\bar{X}$ and $\bar{Y}$ in log (CFU/mI); (-): not determined or precised.

nant une forte proportion de bactéries lactiques dans la flore totale.

\section{DISCUSSION ET CONCLUSION}

Conformément aux études précédentes (Ginn et al, 1984; Dasen et Duboz, 1985; Hughes et Sutherland, 1987; Bishop et Juan, 1988), la technique du film sec réhydratable (Petrifilm SM) présente une relation très étroite avec les techniques de référence pour la numération de la flore totale du lait cru. La précision d'estimation obtenue au cours de notre essai est sensiblement meilleure que celle donnée par Dasen et Duboz (1985); de plus, nous n'avons observé sur film $\mathrm{sec}$ réhydratable aucune sous-estimation des nombres d'UFC/ml que les auteurs précités avaient constatée et attribuée à une inhibition par le TTC de certaines souches bactériennes. Deux raisons principales peuvent expliquer ces différences : d'une part, les améliorations techniques apportées au Petrifilm, avec en particulier les modifications de composition du milieu de culture (D Augusto, comm pers) et d'autre part, la nature de la flore des laits analysés. En effet, notre essai a concerné des échantillons de lait de citernes qui, en raison de leur conservation prolongée à basse température, possèdent souvent une flore plus homogène que les laits prélevés à la ferme.

De même, cette technique du film sec réhydratable (Petrifilm VRB) permet d'estimer avec une précision satisfaisante les nombres de coliformes du lait cru, avec cependant une sous-estimation significative de ces nombres. Cette sous-estimation avait d'ailleurs été notée également par Branger (1987). Celle-ci est assez facile à expliquer; en effet, Nelson et al (1984) ont montré que $91 \%$ des souches productrices de gaz sur Petrifilm peuvent être identifiées comme bactéries coliformes mais que $45 \%$ des souches atypiques (non pro- 


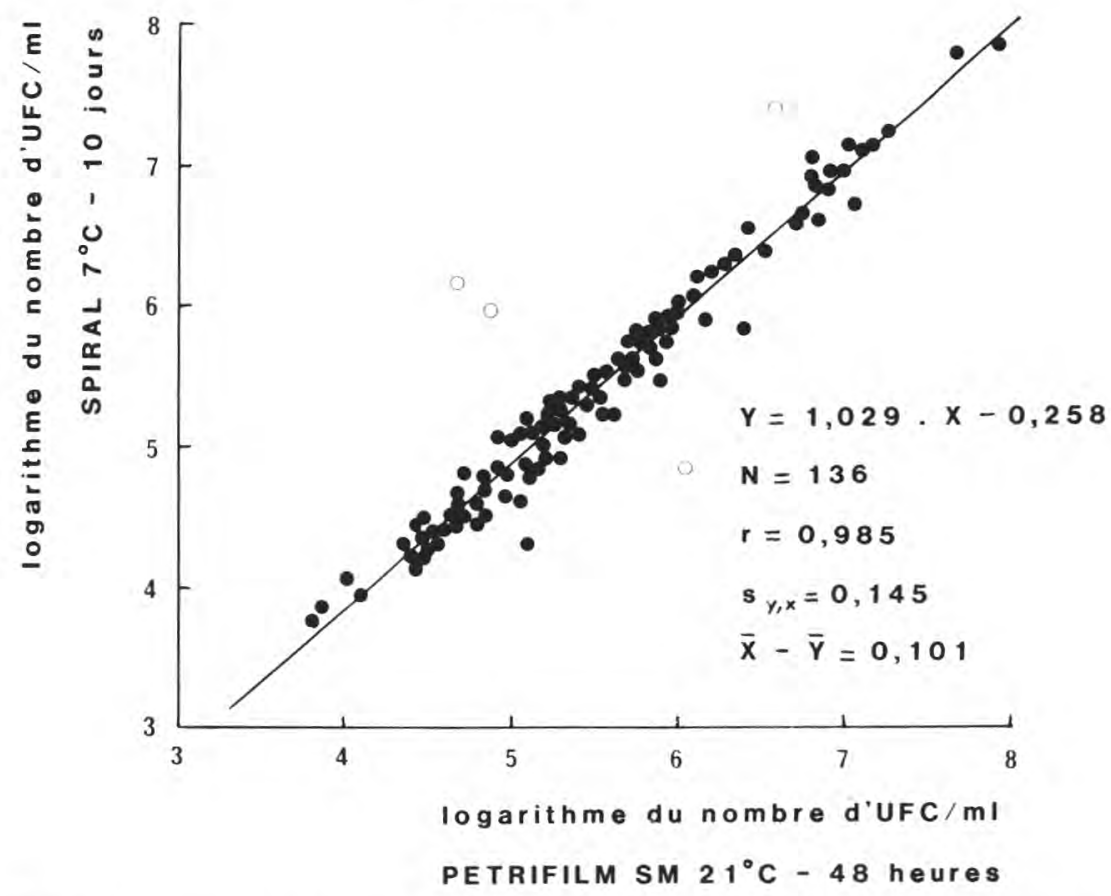

Fig 3. Relation entre les logarithmes décinaux des nombres de bactéries psychrotrophes obtenus selon la technique de référence $\left(7^{\circ} \mathrm{C}\right.$ pendant $\left.10 \mathrm{j}\right)$ et les logarithmes décimaux de ceux obtenus sur film sec réhydratable (Petrifilm SM) après $48 \mathrm{~h}$ d'incubation à $21^{\circ} \mathrm{C}$. L'équation de la droite de calibrage a été calculée après élimination de 4 échantillons $(O)$ qui s'écartent de plus de 3 écarts types résiduels de cette droite.

Relationship between the logarithms of the numbers of psychrotrophic micro-organisms obtained with the reference technique $\left(7^{\circ} \mathrm{C}\right.$ during $\left.10 \mathrm{~d}\right)$ or with the dry rehydratable method (Petrifilm SM) after an incubation of $48 \mathrm{~h}$ at $21^{\circ} \mathrm{C}$.

ductrices de gaz) sont également des souches de bactéries coliformes; à titre de comparaison, d'après ces mêmes auteurs, ces pourcentages sont respectivement de 92 et $3 \%$ pour la méthode de référence sur VRBA. L'allongement de la durée d'incubation au cours de notre essai n'a, semble-til, pas permis d'améliorer la détection des colonies faibles productrices de gaz. D'autre part, la sous-estimation des nombres de bactéries coliformes par la technique du film sec réhydratable peut également être liée en partie au fait que nous n'avons pas recouvert le milieu
VRBA d'une double couche. En effet, Richard et al (1988) ont noté, pour quelques échantillons, la croissance de colonies lactose-positives identifiables à des Vibrionacées sur VRBA sans double couche.

Enfin, notre étude confirme que la technique rapide de numération de la flore psychrotrophe du lait cru décrite par Oliveria et Parmelee (1976) peut être utilisée avec les films secs réhydratables à la place de la technique de référence. La précision d'estimation de cette technique au cours de notre essai est, en effet, très proche de 
celle observée pour la numération de la flore totale. II n'est pas sûr que cette méthode puisse être appliquée avec une précision aussi satisfaisante sur des populations d'échantillons différentes, comme par exemple sur des laits prélevés aussitôt après la traite ou dans les tanks de ferme. En effet, les laits de citernes de ramassage sunt des laits de grand mélange et ont déjà subi une conservation prolongée à basse température qui favorise la prolifération d'un seul groupe de microorganismes : les Pseudomonas psychrotrophes (Richard, 1981); à l'inverse, les laits prélevés à la ferme possèdent généralement une flore de nature plus hétérogène en fonction de leurs conditions de production et de conservation (Thomas et al, 1962; Twomey et Crawley, 1968; Richard, 1981 et 1983). Or, nous avons observé que la nature de la flore des échantillons peut avoir un rôle très important sur la relation entre la technique de référence et celle d'Oliveria et Parmelee (1976); en particulier, la température de $21^{\circ} \mathrm{C}$ ne semble pas inhiber la croissance de certaines bactéries lactiques.

\section{REMERCIEMENTS}

Ce travail a reçu le soutien financier de la Direction générale de l'alimentation du ministre de l'Agriculture (Contrat DGAL nR88/11).

\section{RÉFÉRENCES}

Anonyme (1985a) Lait et produits laitiers. Dénombrement des coliformes. Technique par comptage des colonies et technique du nombre le plus probable à $30^{\circ} \mathrm{C}$. Norme FIL $73 \mathrm{~A}$

Anonyme (1985b) Milk. Definition and Evaluation of the Overall Accuracy of Indirect Methods of Milk Analysis. Application to Calibration Procedure and Quality Control in Dairy Laboratory. Norme provisoire FIL-IDF 128
Bishop JR, Juan JY (1988) Improved methods for quality assessment of raw milk. $J$ Food Prot 51, 955-957

Branger A (1987) Étude du Petrifilm VRB 3M pour la numération des coliformes dans les produits laitiers. Tech Lait 1025, 27-29

Campbell JE, Gilchrist JE (1973) Spiral plate technique for counting bacteria in milk and other foods. Dev Ind Microbiol 14, 95-102

Dasen A, Duboz G (1985) Un Petrifilm pour la détermination de la flore totale du lait cru. Rev Lait Fr 443, 28-30

Donnelly CB, Gilchrist JE, Peeler JT, Campbell JE (1976) Spiral plate count method for the examination of raw and pasteurized milk. Appl Environ Microbiol 32, 21-27

Gilchrist JE, Campbell JE, Donnelly CB, Peeler JT, Delaney JM (1973) Spiral plate method for bacterial determination. Appl Microbiol 25, 244-252

Ginn RE, Packard VS, Fox TL (1984) Evaluation of the $3 \mathrm{M}$ dry medium culture plate (Petrifilm SM) method for determining numbers of bacteria in raw milk. $J$ Food Prot 47, 753-755

Ginn RE, Packard VS, Fox TL (1986) Enumeration of total bacteria and coliforms in milk by rehydratable film methods : collaborative study. J Assoc Off Anal Chem 69, 527-531

Hughes D, Sutherland PS (1987) Evaluation of Petrifilm SM for determining bacteriological counts on raw milk by comparison with the standard plate count and micropipette count techniques. Aust J Dairy Technol 42, 59-61

Jarvis B, Lach VH, Wood JM (1977) Evaluation of the spiral plate maker for the enumeration of microorganisms in foods. J Appl Microbiol $43,149-157$

Nelson CL, Fox TL, Busta FF (1984) Evaluation of dry medium film (Petrifilm VRB) for coliform enumeration. J Food Prot 47, 520-525

Oliveria JS, Parmelee CE (1976) Rapid enumeration of psychrotrophic bacteria in raw milk and pasteurized milk. J Milk Food Technol 39, 269-272

Peeler JT, Gilchrist JE, Donnelly CB, Campbell JE (1977) A collaborative study of the Spiral plate method for examining milk samples. $J$ Food Prot 40, 462-464

Piton C, Grappin R (1990) A model for statistical evaluation of precision parameters of microbiological methods: application to the dry re- 
hydratable film methods and the IDF reference methods for the enumeration of total aerobic mesophilic flora and coliforms in raw milk. J Assoc Off Anal Chem (submitted)

Richard J (1980) Influence de l'agitation du lait cru sur les résultats de dénombrement de sa flore totale à l'aide d'une anse calibrée. Lait 60, 211-225

Richard J (1981) Influence de diverses méthodes de nettoyage des machines à traire sur la «qualité de conservation" du lait cru à basse température. Lait 61, 354-369

Richard J (1983) Nature de la flore microbienne dominante et sous-dominante des laits crus très pollués. Lait $63,148-170$

Richard J, Krebs C, Walter J, Grappin R (1981) Comparaison de divers modes d'agitation des échantillons de lait cru en vue de la numération de sa flore totale suivant la méthode Thompson. Lait 61, 250-260

Richard J, Piton C, Gratadoux JJ (1988) Nature des colonies se développant à la surface du milieu Violet Rouge Bile Lactose Agar (VRB) ensemencé avec du lait cru. Validité des dénombrements des colonies lactose-positives et des colonies lactose-négatives se développant sur ce milieu. Lait 68, 311-322

Thomas SB, Hobson PM, Bird ER, King KP, Druce RG, Cox DR (1962) The microflora of raw milk as determined by plating on yeastrel-milk agar incubated at $30^{\circ} \mathrm{C}$. J Appl BaCteriol 25, 107-115

Twomey A, Crawley WE (1968) The microflora of raw milk. NZJ Dairy Technol 3, 44-48 